\title{
The development of a mobile monitoring system for agricultural object
}

\author{
Sergey Konev ${ }^{1}$, Miroslav Vernezi ${ }^{1}$, Daniil Donskoy ${ }^{1}$, and Alexander Lukyanov ${ }^{1}$ \\ ${ }^{1}$ Don State Technical University, Chair "Production Process Automation”, 344000, Gagarin square, Rostov-on-Don, Russia
}

\begin{abstract}
The article studies the questions of system development for mobile control of data received through wireless networks. For practical implementation, the task has been chosen of control, visualization and saving measurable values received through Bluetooth connection in devices controlled by Android OS. The goal of using such a system may be remote control of temperature, pressure, and other measurable values.
\end{abstract}

\section{Introduction}

The task of remote control of temperature, pressure, and humidity is relevant for many industrial and laboratory applications, for example, in the field of thermal processing, in drying equipment, in the food industry, in tasks of maintenance of a micro-climate of premises for growing plants, etc. Conventional systems of remote monitoring are based on the use of wire systems and PC. Such systems have significant disadvantages: they are bulky, and inconvenient to service and reconfigure. Implementation of wireless technologies in this field enables one to refuse to be bound to a certain stationary work place, and to increase mobility thanks to the use of mobile and wearable devices in the control system as a human-computer interface tool, providing additional convenience for operating personnel and increasing its efficiency [1].

\section{System architecture}

To implement a designed system, it is necessary to select development tools. Based on the task and functions of the system being developed, the following tools can be identified, which are necessary for microclimate control system development:

1. Sensor for temperature measurement

2. Controller for the collection and processing of measured data

3. Device for data transfer to the device for monitoring of readings

4. Client device for temperature monitoring, storage, and visualization of data

\subsection{Temperature sensor}

DHT22 digital temperature and sensor manufactured by Adafruit Industries is used as a measuring device. The sensor consists of a capacitive humidity sensor, a thermistor and contains an ADC for converting analogue values of humidity and temperature. Determination of humidity - from 0 to $100 \%$ with $2 \%$ accuracy. Determination of temperature - from -40 to $120{ }^{\circ} \mathrm{C}$ with an accuracy of $\pm 0.5^{\circ} \mathrm{C}$. Sampling frequency - not exceeding $0.5 \mathrm{~Hz}$ (no more than 2 times per second) [2].

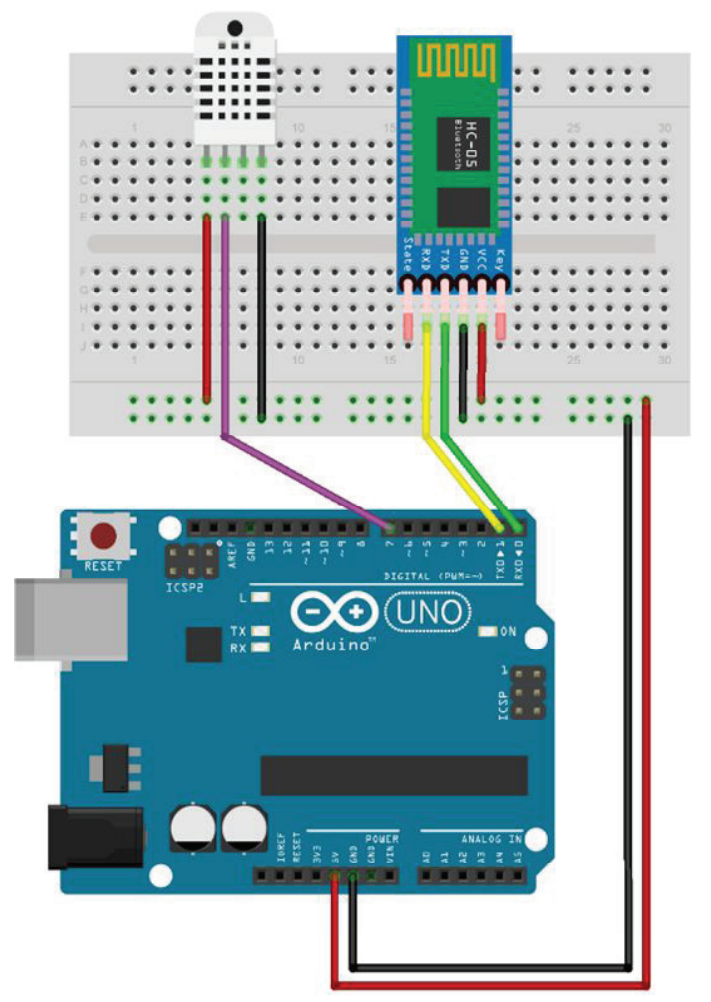

Fig. 1. Connection diagram of the Arduino controller with DHT22 sensor and HC06 module.

\footnotetext{
*Corresponding author: lex1998@rambler.ru
} 


\subsection{Controller for data collection and processing}

As the device for the primary collection of sensor readings, the most popular controller of the Arduino family is used - Arduino UNO, which continues the very first controller product line. The Arduino Uno controller can be used to create systems for receiving and processing signals from various digital and analogue sensors, and controlling various actuators.

The Arduino Uno controller can be used to create devices and systems for receiving and processing signals from various digital and analogue sensors that can be connected to it, and controlling various actuators [3]



Fig. 2. System operation algorithm.

\subsection{Wireless communication protocol and module}

Bluetooth protocol is used as the technology for wireless communication between devices. This protocol uses a low power radio interface and was developed as a replacement for existing serial cable interfaces in consumer electronics and industrial applications[4].

Bluetooth technology is a flexible and easy-to-use wireless solution. Operating in the same $2.4 \mathrm{GHz}$ free ISM band (industrial, scientific and medical bands) as other wireless protocols, Bluetooth has optimal characteristics in terms of requirements for stability, reliability, and interaction with wireless LANs, specified by the industry [5].

Data transfer through Bluetooth between the controller and Android-based device is conducted with the help of a connectable external Bluetooth module HC06 manufactured by Olimex. It is an open-frame monoboard module intended for surface mounting. The board provides support for the Bluetooth 2.0 EDR protocol stack at the hardware level. Built-in flash memory capacity for storing firmware and setup settings is $1 \mathrm{MB}$. Maximum signal range is $30 \mathrm{~m}$ [6]. Figure 1 shows the connection diagram of the Arduino controller with temperature sensor and Bluetooth module. 


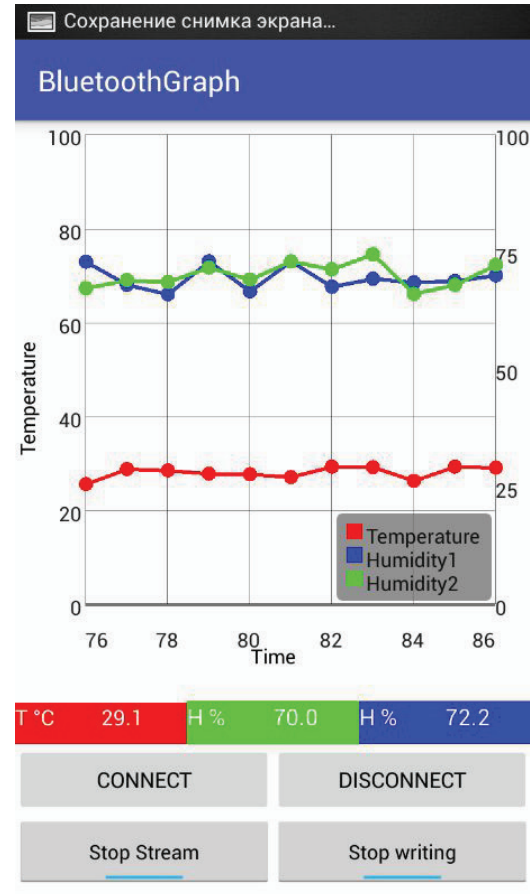

a)

50

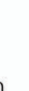

$\emptyset \square \stackrel{\square}{\square}$

Paired Devices

SPP-CA (Paired)

00:BA:55:56:E3:19

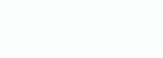

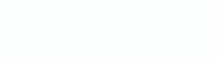

\section{.}

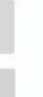


on an Android-based mobile device, for building real time graphs based on data transferred by an Arduino controller via a Bluetooth connection is presented.

\section{References}

1. R. Nilsson, Industrial wireless: Bluetooth can be robust, easy to use (2013) Retrieved: September 22, 2017

2. DHT22 Temperature and humidity sensor. Retrieved September 21, 2017

3. Microcontroller Arduino UNO Retrieved September 22, 2017

4. D.A. Gratton, The Handbook of Personal Area Networking Technologies and Protocols. (Cambridge University Press, Cambridge, 2013)

5. Bluetooth Core Specification v.5.0. Retrieved September 19, 2017

6. Bluetooth-Serial module HC-06. Retrieved September 19, 2017

7. S. Conder, L. Darcey, Android Wireless Application Development. Second edition. Addison-Wesley, (2011)

8. Android's Bluetooth API developer guide. Retrieved September 19, 2017

9. GraphView - graph-plotting library for Android. Retrieved September 19, 2017 